\section{$\dagger 864$}

ISOLATED THROMBOCYTOPENIA FOLLOWING ALLOGENEIC BONE MARROW TRANSPLANTATION. LR First, BR Smith, JM Lipton, School, Departments of Medicine and Pediatrics, Boston, MA. Isolated thrombocytopenia following bone marrow transplantation was investigated in 65 fully grafted patients (pts) surviving 60 days post transplant. 24 pts $(37 \%)$ developed this complication, which occurred most frequently in pts receiving pre-tx preparation with total body irradiation or busulfan. Two distinct syndromes were identified: (1) transient thrombocytopenia established by day +40 but with subsequent resolution by day +90 . Three of these cases were associated with trimethoprim-sulfamethoxazole therapy. (2) chron ic thrombocytopenia (15 pts) in which a plt count $>100,000$ was not achieved at any time during the first 4 mo post-tx despite the simultaneous presence of normal granulocyte and reticulocyte counts. No association with drug therapy was detected. While the transient syndrome did not adversely affect prognosis, the chronic syndrome carried a high mortality (21\% actuarial survival at 1000 days post-tx compared to $67 \%$ for all other pts $p(0.01)$. The mortality was not due to bleeding but rather to a high association with both severe acute (grade III-IV) graft versus host disease and chronic GVHD. We conclude that isolated thrombocytopenia represents a significant complication of bone marrow transplantation particularly in pts receiving hematopoietic ablative preparatory regimens and that it is the chronic, and not the transient, thrombocytopenic syndrome that adversely affects patient prognosis as a manifestation of GVHD.
867 AND

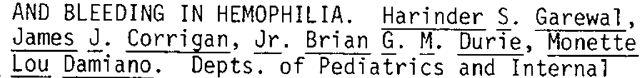
Jeter, Mary Lou Damiano. Depts. of Pedia trics and Internal Danazol has been reported to raise several plasma proteins. Danazol was given orally at $600 \mathrm{mg} /$ day in 3 divided doses to 6 hemophiliacs for 8-14 weeks. Factor levels, APTT and other laboratory parameters were measured every 2 weeks. Five patients had classic hemophilia ( 1 mild-moderate, 3 severe, I with inhibitor) and 1 had Christmas disease. All patients showed a significant decrease an APTT beginning with the first measurement (2 weeks) and persisting till the drug was discontinued. However, a corresponding increase in the deficient factor activity could not be consistently demonstrated. Typical results for a severe classic hemophiliac are shown.

APTT(secs)
$\frac{\text { Baseline On Danazol (mean) }}{90} \frac{\text { Baseline On Danazol (mean) }}{1.1}$
Despite the shortened APTT bleeding episodes continued in the severe hemophiliacs and the patient with Christmas disease. In 4 patients bleeding appeared to either increase in severity or change in pattern. In 2 patients bleeding did not respond to their usual factor infusions, but responded to discontinuation of danazol and further factor replacement. In 3 patients the drug was discontinued because of bleeding. These results differ from those recently reported. Increased fibrinolytic activity may be responsible for the al tered bleeding.

865 ISOLATED CHOROIDAL RELAPSE DURING COMPLETE REMISSION M. H. MAOR, L. P. STEAHLY, R. A. TANG, \&.G. TAYLOR,

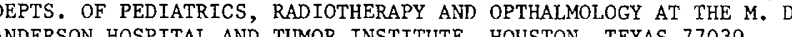
Leukemic opthalmopathy is of tem diagnosis of ocular leukemia is much less common and has been reported associated with central nervous system (CNS) \&/or multiple bone marrow (BM) relapses. Leukemia was diagnosed in the chorold of a patient ( $p t$ ) with ALL in complete remission (CR) during therapy. An 11 yo Black girl had null cell ALL, CaLla and Ia positive. The CNS had no evidence of disease. Initial therapy was "standard treatment". Six mos after diagnosis the pt complained of decreased visual acuity and was found to have perivascular leukemic deposition in the ocular fundi. Fluorescein angiography and ultrasonography of the globe verified infiltration with bilateral thickening of the chorold layer. BM and spinal fluid examination were normal. Cranial radiotherapy (XRT), was administered to the whole brain, including the posterior pole of the eyes. The pt continued systemic therapy. In the 9 mos since XRT: Vision has improved, fluorescein angiography shows residual pigmentary changes, ultrasonography reveals normalization of the choroid, \& the pt remains in CR. Leukemic relapse of the choroid is a dangerous sanctuary not accessible to biopsy. Standard opthalmoscopic examination provides an adequate view of only the posterior pole; without dilation, peripheral lesions cannot be seen. Since peripheral fundal lesions are generally asymptomatic, opthalmologic consultation is indicated at diagnosis, periodically throughout treatment, \& at termination of therapy for ALL.

866 ANTIPROLIFERATIVE PROPERTIES OF GENE-CLONED ALPHA INTERFERON IN ACUTE LEUKEMIA. Melvin H. Freedman, Toronto, Hosp for Sick Children, Divs of Hematology, Infectious Diseases, and Immunology, Toronto, Canada.

The properties of recombinant-DNA human leukocyte interferon (HuINF $\alpha 2$, Schering-Plough Corp) were studied in children with advanced acute leukemia (1 each of AML, T-cel1 ALL, and non-Tnon-B ALL). When marrow blasts were exposed to IFN in vitro, there was a marked rise in cellular 2-5A synthetase comparable to control marrow, indicating full expression of IFN receptor sites on these cells as well as their ability to react metabolically. IFN also induced a striking dose-responsive decline in leukemic blast progenitor colony formation and on blast self-renewal in vitro, confirming its antiproliferative effect. When the patients were given high-dose IFN alone (up to $\left.100 \times 10^{6} \mathrm{u} / \mathrm{m}^{2} \mathrm{I} . V_{.}\right)$, blast cytoreduction was seen in peripheral blood in ail, and in marrow of the AML. Also after IFN was given, marrow and peripheral blood cells demonstrated elevated 2-5A synthetase activity in vivo, similar to the effect seen in vitro. No modulation of leukemic cell markers was seen following in vitro or in vivo treatment with IFN, implying that cytoreduction was not linked to blast differentiation. These studies suggest that this subtype of gene-cloned IFN has antileukemic properties, and indicates the possibilities for IFN as an adjunctive form of therapy in childhood leukemia.
IMMUNOLOGICAL EVALUATION OF PATIENTS WITH HEMOPHILIA-

868 R. Gera, Z. Jin, R. Cleveland, D. Murray, R.Kulkarni, E. Romond, and D.B. Kaufman. Department of Pediatrics/Human Development, East Lansing, MI and Regional Great Lake Red Cross Blood Center, Lansing, MI.

AIDS is characterized by defects in the cell mediated immune system resulting in unusual infections, neoplasms and immune phenomena. Patients with hemophilia A (HemA) treated with lyophilized commercial FVIII (Lyoph-c) are at risk of developing AIDS since many have AIDS like defects. We have studied 41 patients with hemophilia and Von Willebrand's disease (VWD): 8 Hem A treated with Lyoph-c, 7 Hem A treated with lyophilized volunteer plasma FVIII (1yoph-v), 8 Hem A with inhibitor (Hem A-inh) and 10 Hem $B$ treated with activated or nonactivated prothrombin complex (PTC a/n) and 8 untreated Hem $A$ and VWD. Overall $41 \%$ had $T$ helper/T supressor ( $\mathrm{OK} \mathrm{T}_{4} / \mathrm{T}_{8}$ ) ratios less than 1.4. Although this defect was most marked in the lyoph-c and lyoph-v group $(8 / 15$ abnormal), a similar defect was observed in Hem A-inh (3/8), Hem $\mathrm{B}(2 / 10)$ and untreated $(4 / 8)$ groups. Only two patients with abnormal. $\mathrm{T} / \mathrm{T} / \mathrm{T}$ had laboratory evidence of ongoing viral activity. Serum Beta-Z-microglobulin $\left(\beta_{2} \mathrm{~m}\right)$ levels were elevated (greater than $2000 \mu \mathrm{g} / \mathrm{L}$ ) in $93 \%$ of treated patients and in only $12.5 \%$ of untreated patients. Lymphocyte blastogenesis and MLC were normal in most of the patients. There was no correlation be tween Tcell markers $\left(\mathrm{T}_{4} / \mathrm{T}_{8} ; \mathrm{T}_{8} \% ; \mathrm{T}_{4} \%\right)$ and amount of factor used. Our results indicate that immunologic abnormalities are present in hemophiliacs \& VWD, and that a11 such patients may be at risk
for developing AIDS. Elevated $\beta_{\mathrm{m}}$ in treated group may reflect chronic antigenic stimulation in these patients.

A REEVALUATION OF THE BLEEDING TIME: J.M. Gerrard, S.J. Israels, M. Cheang, A.J. Bishop, H.L. Rayner, N.L. Kobrinsky, M.L. Schroeder and E.D. Israels Departments of Pediatrics, Medicine and Computer Science, University of Manitoba, Winnipeg, Manitoba, Canada.

A multivariate analysis of results of 512 individuals, (more than half less than 20 years of age) referred to the University of Manitoba Coagulation Laboratory, was performed to assess the relationship of age, sex, and various coagulation parameters to the length of the bleeding time (BT). Patient age (children had longer BTs than adults, even with other factors normal), platele aggregation to collagen and epinephrine, platelet adhesion (ADH) and prothrombin consumption (PC) emerged as important and independent variables $(p<0.01)$. Controlling for other variables, including von Willebrand (vW) $\mathrm{Ag}$ and ristocetin cofactor (RC), did not change the correlation of $\mathrm{ADH}$ and $\mathrm{BT}$, but decreased that between PC and BT. Where vWAg, VWRC and factor VIII were all less than $120 \%$, there was a weak negative correlation between the length of the BT and vWAg or vWRC. In contrast, where any one of these three parameters was $120 \%$ or greater, there was a weak of these three parameters was $120 \%$ or greater, there was a weak pos-
itive correlation between vWAg or vWRC and the BT. However, the itive correlation between vWAg or vWRC and the BT. However, the
mean BT of a11 (8) patients with vWAg or vWRC less than $55 \%$, nor-
mal aggregation and PC greater than $80 \%$, was in mal aggregation and $\mathrm{PC}$ greater than $80 \%$, was in the normal range $(7.56 \mathrm{~min})$. The view that vWAg level, or activity as assessed by VWRC, is, by itself, a major determinant of the length of the BT should be revised. Platelet adhesion, independent of factor level (VWAg) or activity (VWRC) appears more important. 